\title{
Primary Seminal Vesicle Abscess: A Diagnostic Dilemma- A Review of Current Literature
}

\author{
Fahim LH Rajiwate ${ }^{1}$, Deepak Bolbandi²
}

\begin{abstract}
Seminal vesicles (SV) are accessory sex organs of male genitourinary (GU) tract, which play a crucial role in male fertility. Primary seminal vesicle abscess (SVA) is a rare pathologic entity with no specific symptoms with very few reported cases in literature, posing a great diagnostic difficulty to the clinicians. Common pathologies of seminal vesicle include cyst, congenital abnormalities and seminal vesicle infection, and abscess, secondary to obstruction and infection of the neighboring organs have been frequently addressed. Cross-sectional imaging advancement has expanded not only our knowledge of GU tract abnormalities but also our treatment approaches. Seminal vesicle abscess is a rare pathological condition and diagnosis is based on clinical and radiological findings. Conservative treatment could be effective in selected cases. In cases refractory to conservative management, surgical decompression can be an option. Transrectal ultrasonography-guided mini-invasive drainage modalities can be proposed successfully.
\end{abstract}

Keywords: Abscess drainage, Magnetic resonance imaging, Seminal vesicle, Transrectal ultrasonography.

The Journal of Medical Sciences (2019): 10.5005/jp-journals-10045-00126

\section{INTRODUCTION}

The seminal vesicles (SVs), which play a major role in male fertility, are part of the three main accessory glands of the male reproductive system, namely, prostate, and bulbourethral glands. Seminal vesicle abscess (SVA) is a rare uropathologic entity. The first case of SVA was reported by Rajfer et al. ${ }^{1}$ Seminal vesicle abscess poses a diagnostic difficulty as symptoms onset resembles to several other diseases of the lower genitourinary (GU) tract. Acute and/or chronic seminal vesiculitis usually secondary to an infection forms an abscess, which also affects the prostate gland. Clinical assessment becomes difficult in the setting of concomitant prostatitis or prostatic abscess, where the prostate gland is enlarged and tender. However, diagnosis is critical due to implications on reproductive health and quality of life.

Clinical presentation secondary to seminal vesicle involvement is highly variable and includes fever, dysuria, recurrent epididymoorchitis, rectal discomfort, constipation. diarrhea, frequency, hematuria, urgency, obstructed flow/urinary retention, abdominal/ flank/pelvic/groin pain, incontinence, hemospermia, indurated cord, inguinal pain, pyuria, and in some rare cases pneumaturia.

The etiology of SVA is not clearly understood, and many predisposing factors have been reported without statistical evidence and includes diabetes mellitus, ${ }^{2}$ HIV-positive status, ${ }^{3}$ alcoholic cirrhosis, ${ }^{4}$ recurrent and unresolved urinary tract infection, 5,6 indwelling Foley catheter, urological instrumentation and procedures, pelvic infection, ${ }^{6}$ renal agenesis, ${ }^{5}$ and associated Wolffian duct abnormalities.

In endemic areas, Mycobacterium tuberculosis (TB) must be considered as a possible etiology of a GU abscess. Seminal vesicle tuberculous involvement is invariably associated with involvement of the prostate and the upper urinary tract (kidneys and ureters). Initially, there is SV enlargement with the loss of convolutions; the resultant abscess undergoes caseation, cavitation, and fibrosis eventually forming a calcified mass. Schistosomiasis caused by Schistosoma haematobium, endemic in Africa and the Middle East commonly affects the GU system. ${ }^{7,8}$ In contrast to tuberculosis in which there is luminal calcification, schistosomiasis has wall
1,2Department of Urology, RajaRajeswari Medical College and Hospital, Bengaluru, Karnataka, India

Corresponding Author: Fahim LH Rajiwate, Department of Urology, RajaRajeswari Medical College and Hospital, Bengaluru, Karnataka, India, Phone: +91 8692004048, e-mail: fahimlhr@hotmail.com

How to cite this article: Rajiwate FLH, Bolbandi D. Primary Seminal Vesicle Abscess: A Diagnostic Dilemma-A Review of Current Literature. J Med Sci 2019;5(3):67-69.

Source of support: Nil

Conflict of interest: None

calcifications as schistosome eggs are deposited into the wall of the SV tissue rather than the lumen that does not resolve with treatment. Diagnostic confirmation can be obtained with bacterial and parasitological study of the semen and urine in a symptomatic patient traveling to an endemic area with SV and vas deferens (VD) calcifications on imaging.

\section{Diagnosis}

As SVs neighbor the bladder base, prostate, rectum, ureters, and peritoneum, presenting symptoms of SV inflammation and abscess, are often varied and overlapping. The symptoms can be fever, irritative urinary symptoms, dysuria, urinary retention, hemospermia, hematuria, rectal discomfort, tenesmus, and perineal or inguinal pain. ${ }^{9}$ Digital rectal examination usually shows an enlarged prostate usually unilateral tender mass higher over the involved seminal vesicle, which is difficult to interpret and misdiagnosed. Ipsilateral spermatic cord induration and perineal tenderness are other clinical findings. An untreated SVA may burst into urethra, perirectal tissues presenting as an ischiorectal abscess, perineum, or even rectum to form a recto-urethral fistula. Hence, clinical diagnosis of primary SVA becomes diffuse and difficult. This leads to the use of several diagnostic modalities including transrectal ultrasonography (TRUS), transabdominal ultrasound (TAU), CT, and magnetic resonance imaging (MRI). ${ }^{2}$ 
Transabdominal ultrasound is used to visualize the presence of abscess, which is characterized as heterogeneous and moderately hypoechoic round lesions with thick walls, septations, and increased vascularity on Doppler scanning. Via transperineal or transrectal approach, puncture and drainage of abscess with minimum morbidity is possible under US guidance. ${ }^{10,11}$ The first to report the role of TRUS in the diagnosis of SVA was by Lee et al. ${ }^{12}$ Realtime imaging showing the extent of the abscess cavity, the image capability into the longitudinal and axial planes, and ability to perform an ultrasound-guided puncture of the lesion for diagnostic and therapeutic purpose are advantages of TRUS over CT and MRI in the diagnosis of SVA.

Contrast-enhanced CT scan has been the most common imaging modality utilized for the diagnosis of SVA. ${ }^{13,14}$ CT characteristics findings of SVA have been described as:

- Unilateral or bilateral seminal vesicle enlargement

- Hypodense areas within the seminal vesicle

- Adjacent fat stranding

- Focal or diffuse thickening of the bladder wall.

On CT scan, the periprostatic inflammatory changes with dilatation of the intraprostatic ejaculatory duct can be misinterpreted as a prostatic abscess.

Seminal vesicle abscess can be verified by MRI. Seminal vesicles are fluid-rich, hence hypointense on $T_{1}$-weighted and hyperintense on $T_{2}$-weighted images. Inflammatory findings of the SVs include enlargement and alteration of signal intensity.

The inflamed seminal vesicle on $T_{1}$-weighted images shows decreased signal intensity as compared to normal, whereas on $T_{2}$-weighted images, the signal intensity increases significantly as compared to normal and is distinctly higher than fat. Magnetic resonance imaging shows their size, number, and invasion of adjacent organs clearly facilitating qualitative and quantitative diagnostic evaluation of prostate and SVA and its differentiation. Due to its higher spatial resolution, better tissue contrast, and multiplanar imaging capabilities, MRI is considered superior to $\mathrm{CT}$ and ultrasound in assessing infection involving prostate and seminal vesicle. ${ }^{15}$

High-resolution and functional MRI studies widely used in patients with prostate carcinoma have also been applied to diagnose SVA. Noninvasive functional MR technique, diffusion-weighted imaging (DWI), has gained recognition as an imaging biomarker for tissue characterization and functional evaluation as it analyses the diffusion of water molecules in vivo. ${ }^{16}$ It has been used to assess the prostate mainly in carcinoma prostate. However, recently, the use of DWI has expanded to the field of diagnosis of deep pelvic abscess. Three-dimensional reconstruction and fusion with T2WI after DWI may further facilitate spatial colocation of anatomical background and functional information obtained from DWI. Therefore, DWI may help to diagnose abscess with higher specificity. In patients who may not be able to receive intravenous contrast agent due to either a history of allergy or renal failure, MRI-DWI may be a stand-alone technique for diagnostic evaluation. With various options available, TRUS and CT remain to be the most frequently described diagnostic examinations in the recent literature. ${ }^{17}$

\section{Treatment Modalities}

Seminal vesicle has an ejaculatory duct that opens into the prostatic urethra; hence, in contrast to other abscess cavities, SV abscess should be able to drain into the prostatic urethra as long as ejaculatory duct remains open. Conservative measures may help to decrease inflammation and further open up a partially obstructed EJD system. Intravenous antibiotic therapy is the first-line therapy in SVA and can be managed conservatively with antibiotics alone. ${ }^{18,19}$ If the patient is not responding clinically to intravenous antibiotics, early drainage of the abscess is indicated. Approach can be transpubic percutaneous or transvesical, transperineal, transurethral, transrectal, or via an open surgical approach. ${ }^{2}$

Ultrasound-guided transperineal or transrectal puncture and aspiration can be performed rapidly, successfully, and easily; however, sometimes the purulent aspirate can be too viscous to be drained adequately via these techniques. ${ }^{12}$ Other authors ${ }^{2,3}$ propose a transurethral drainage. Frye ${ }^{2}$ proposed the injection of methylene blue dye via vasotomies to facilitate the identification of the abscess cavities and guide the depth of transurethral drainage. Major surgical interventions, such as transvesical, retrovesical, or perineal surgery have significant associated morbidity affecting quality of life and poor health outcomes.

\section{ConCLUSION}

The SVs form an integral part of the three main accessory glands of the male reproductive system, namely, prostate, and bulbourethral glands. Seminal vesicle abscess is a rare pathology with no specific symptoms.

Often, pathology involving SVs also affects the nearby prostate and, in many instances, arises initially within the prostate gland, resulting in pathology being masked or ignored within the SV posing great clinical diagnostic difficulties. Nevertheless, with advances in cross-sectional techniques of prostate imaging, discrete lesions of the SV can be diagnosed with precision. Transrectal ultrasonography and CT scan are the imaging modalities of choice for diagnosis.

Conservative treatment could be effective in selected cases while surgical decompression should be considered in refractory cases treated conservatively. Transrectal ultrasonography-guided mini invasive drainage modalities can be proposed successfully.

\section{References}

1. Rajfer J, Eggleston JC, Sanders RC, et al. Fever and prostatic mass in a young man. J Urol 1978;119(4):555-558. DOI: 10.1016/S00225347(17)57546-1.

2. Frye $\mathrm{K}$, Loughlin K. Successful transurethral drainage of bilateral seminal vesicle abscesses. J Urol 1988;139(6):1323-1324. DOI: 10.1016/ S0022-5347(17)42910-7.

3. Jensen $\mathrm{KH}$, Lundvall L. Peritonitis caused by abscess in the seminal vesicle. Case report. Acta Chir Scand 1988;154(3):245.

4. Bonekamp S, Jolepalem P, Lazo M, et al. Hepatocellular carcinoma: response to TACE assessed with semiautomated volumetric and functional analysis of diffusion-weighted and contrast-enhanced MR imaging data. Radiology 2011;260(3):752-761. DOI: 10.1148/ radiol.11102330.

5. Oto A, Schmid-Tannwald C, Agrawal G, et al. Diffusion-weighted MR imaging of abdominopelvic abscesses. Emerg Radiol 2011;18(6): 515-524. DOI: 10.1007/s10140-011-0976-1.

6. Park HJ, Kim SH, Jang KM, et al. Differentiating hepatic abscess from malignant mimickers: value of diffusion-weighted imaging with an emphasis on the periphery of the lesion. J Magn Reson Imaging 2013;38(6):1333-1341. DOI: 10.1002/jmri.24112.

7. Centers for Disease Control and Prevention. Parsites-Schistosomiasis: Epidemiology and Risk Factors. [Last accessed on 2012 Jul 11]. Available from: http://www.cdc.gov/parasites/schistosomiasis/epi. html. 
8. Shebel HM, Elsayes KM, Abou El Atta HM, et al. Genitourinary schistosomiasis: Life cycle and radiologic-pathologic findings. Radiographics 2012;32(4):1031-1046. DOI: 10.1148/rg.324115162.

9. Fox CW, Vaccaro JA, Kiesling VJ, et al. Seminal vesicle abscess: the use of computerized coaxial tomography for diagnosis and therapy. J Urol 1998;139(2):384-385. DOI: 10.1016/S0022-5347(17)42427-X.

10. Porfyris $\mathrm{O}$, Kalomoiris P. Prostatic abscess: case report and review of the literature. Arch Ital Urol Androl 2013;85(3):154-156. DOI: 10.4081/ aiua.2013.3.154.

11. Ruíz Plazas X, Alonso Rodríguez D, Fernández Barranco L, et al. Rectoprostatic fistula: unusual presentation of a prostatic abscess. Arch Esp Urol 2011;64(1):59-61.

12. Lee SB, Lee F, Solomon MH, et al. Seminal vesicle abscess: diagnosis by transrectal ultrasound. J Clin Ultrasound 1986;14(7):546-549. DOI: 10.1002/jcu.1870140709.

13. Zagoria RJ, Papanicolaou N, Pfister RC, et al. Seminal vesicle abscess after vasectomy: evaluation by transrectal sonography and CT. AJR Am J Roentgenol 1987;149(1):137-138. DOI: 10.2214/ajr.149. 1.137.
14. King BF, Hattery RR, Lieber MM, et al. Seminal vesicle imaging. Radiographics 1989;9(4):653-676. DOI: 10.1148/ radiographics.9.4.2667051.

15. Aphinives $C$, Pacheerat $K$, Chaiyakum J, et al. Prostatic abscesses: radiographic findings and treatment. J Med Assoc Thai 2004;87(7): 810-815.

16. Giannarini G, Nguyen DP, Thalmann GN, et al. Diffusion-weighted magnetic resonance imaging detects local recurrence after radical prostatectomy: initial experience. Eur Urol 2012;61(3):616-620. DOI: 10.1016/j.eururo.2011.11.030.

17. Saha $S$, Wright $G$, Arulampalam T, et al. An unusual groin mass. Seminal vesicle abscess: a case report. Cases J 2009;2:6531. DOI: 10.1186/17571626-2-6531.

18. Kang YS, Fishman EK, Kuhlman JE, et al. Seminal vesicle abscesses: spectrum of computed tomographic findings. Urol Radiol 1989;11(3):182-185. DOI: 10.1007/BF02926510.

19. Kennelly MJ, Oesterling JE. Conservative management of a seminal vesicle abscess. J Urol 1989;141(6):1432-1433. DOI: 10.1016/S00225347(17)41337-1. 